University of Wollongong

Research Online

Faculty of Engineering and Information

Faculty of Engineering and Information

Sciences - Papers: Part A

Sciences

$1-1-2013$

\title{
A novel formulation for optimum conceptual design of buildings of rectangular shapes
}

\author{
Pezhman Sharafi \\ University of Wollongong, psharafi@uow.edu.au \\ Muhammad Hadi \\ University of Wollongong, mhadi@uow.edu.au
}

Lip H. Teh

University of Wollongong, Iteh@uow.edu.au

Follow this and additional works at: https://ro.uow.edu.au/eispapers

Part of the Engineering Commons, and the Science and Technology Studies Commons

Research Online is the open access institutional repository for the University of Wollongong. For further information contact the UOW Library: research-pubs@uow.edu.au 


\title{
A novel formulation for optimum conceptual design of buildings of rectangular shapes
}

\begin{abstract}
In many optimum design cases, we look for a set of design variables selected from a given list which assure the optimum of objective function together with satisfaction of the constraints. That is, optimum conceptual design mostly consists of selecting the best combination of a finite number of structural elements and the available parameters. In this paper a novel technique is presented for the conceptual design optimization of framed buildings with a rectangular plan. The method supports all the buildings with grid pattern column layout. To that end, we take advantage of the knapsack problem as a basic applied combinatorial optimization problem. The objective is to find a rectangular column layout of maximum profit for the frames that also satisfy the imposed geometric constraints. The method is presented in a general form and is not confined to optimization under a certain type of action effect and/ or geometrical constraints. It gives the methodology the ability of being formulated for various objective functions and constraints and different structural systems. Civil-Comp Press, 2013.
\end{abstract}

\section{Keywords}

optimum, conceptual, rectangular, design, shapes, formulation, novel, buildings

\section{Disciplines}

Engineering | Science and Technology Studies

\section{Publication Details}

Sharafi, P., Hadi, M. N. \& Teh, L. H. (2013). A novel formulation for optimum conceptual design of buildings of rectangular shapes. Proceedings of the Fourteenth International Conference on Civil, Structural And Environmental Engineering Computing United Kingdom: Civil-Comp Press. 


\title{
A Novel Formulation for Optimum Conceptual Design of Buildings of Orthogonal Shapes
}

\author{
P. Sharafi*1 ${ }^{* 1}$ Lip. H. Teh ${ }^{2}$, Muhammad N. S. Hadi ${ }^{3}$ \\ School of Civil, Mining and Environmental Engineering, University of Wollongong, \\ Northfields Avenue, Wollongong, NSW 2522, Australia
}

\begin{abstract}
This paper presents a description of a new methodology for the optimum preliminary layout design of reinforced concrete multi-span beams considering the relevant cost elements. First, a new objective function is presented, as an alternative to traditional cost functions for reinforced concrete beams. The ability of being easily employed in layout optimization problems, gives the new cost function a distinct advantage over its alternatives. Examples are included to illustrate the performance of the new methodology.
\end{abstract}

Keywords: cost optimization; layout optimization; reinforced concrete; multi-span beam; Ant colony optimization.

\section{Introduction}

The design process can be divided into four stages: the formulation of functional requirements stage, the conceptual (preliminary) design stage, the optimization stage and the detailing stage. An iterative procedure for the four stages is often required before achieving the final solution. In what is named optimal design, the required structural behaviour together with the design loads and geometrical constraints are initially specified and then, the cost or the objective function is defined. In a comprehensive structural optimization process, selecting an appropriate preliminary geometric layout design of structures is of great importance, as it influences all the subsequent stages of the design procedure. The aim of this computational effort is to

\footnotetext{
${ }^{1}$ Research Associate/PhD candidate, School of Civil, Mining and Envir. Eng., Univ. of Wollongong, Wollongong, NSW, Australia.*(Corresponding author).Email address: ps170@uow.edu.au

${ }^{3}$ Senior Lecturer, School of Civil, Mining and Envir. Eng., Univ. of Wollongong, Wollongong, NSW, Australia

${ }^{2}$ Assoc. Professor, School of Civil, Mining and Envir. Eng., Univ. of Wollongong, Wollongong, NSW, Australia.
} 
determine the preliminary geometry so that the desired behaviour is achieved at the lowest possible cost. The outcome can be considered as an optimum starting point, in a comprehensive optimization design process of reinforced concrete continuous beams.

Preliminary design, as the earliest phase of the design process, commences with a set of initial concepts. Designers at this early stage must understand the many factors affecting the project being designed, including account for efficiency, construction cost, operation cost, quality and comfort of the built project, and the potential for revenue generation. Significant complexity comes from the need to determine the relative benefits of all of these various quantities and qualities [1]. In fact, generally there is not a single layout design solution having optimal performance with respect to all requirements because the objective criteria are often conflicting, and designers must evaluate different competing criteria with the view to achieve a good compromise design. That is, the selection of a suitable layout design involves making informed subjective compromises between conflicting objective criteria.

The phase of preliminary layout design is usually carried out according to the architectural requirements without considering the relative costs of concrete, reinforcing steel, formwork or other relevant costs. In fact, the phases of the detailed design and optimization, only deal with a predefined layout plan and the effects of the preliminary layout design on the total costs are mainly neglected. Albeit, designers often are intended to spend most of their working time on the detailed design phase, where the scope for improvement is much less [2]. On the other hand, in contrast to steel structures, where the cost optimization can, more or less, be formulated as a weight minimization problem; for concrete structures, the optimization problem needs to be formulated as a cost minimization problem; because different materials are involved. In practice, a minimum weight design may not lead to a minimum cost design in concrete structures. Ideally, the optimization problem should be considered in terms of costs of materials, fabrication, erection, maintenance, and disassembling the structure at the end of its life cycle as the cost elements.

A number of methods have been developed as general methods for the layout optimization of structures [3-5]. In literature, topology or layout optimization methods rarely consider the cost factors and the objective function is optimized regardless of the involving cost elements. Therefore the topology optimization methods may result in a sub-optimal solution.

Sharafi et al.[6-11], Govindaraj and Ramasamy [12] have attempted to make use of evolutionary methods, to deal with the problem in a discretized form. Muc and Gurba [13] described the concept of using Genetic Algorithm in layout optimization of composite structures. Wang and Liu [14] described a methodology for optimizing both weight and cost for composite structures. Hadi [15] employed a Neural Network (NN) method to deal with the cost optimization of RC beams. Recently, using new heuristics, some different methods have been employed for the layout optimization of structures by Nimtawat and Nanakorn [16, 17], Zhu et al. [18], Shaw et al. [19]. Zou [20] described a multiobjective life-cost optimization approach for topologically predefined reinforced concrete frames. Liu and Qiao [21] presented a 
technique for topology optimization of structures with different tensile and compressive properties in layout design of bridges.

Nevertheless, the applications of these methods are limited to either cost optimization of structures with predefined shapes or layout optimization of structures without considering the relevant costs. Therefore, there is a need to study topology optimization methods that can take cost into account. This study describes a developed approach for optimization of preliminary layout that considers all cost elements and is applicable for multi-span RC beams.

At first, a new cost function is proposed to deal with the cost optimization problem of rectangular RC beams, which can be used in layout optimization of multi-span $\mathrm{RC}$ beams as well as considering the cross-sectional action effects. Then, some numerical examples demonstrate the methodology.

\section{Problem Definition}

When designing concrete structures, the designer is faced with four classes of design variables. The first class is material design variables such as the type of concrete to be used. The second class is topological design variables such as the number of spans in a frame. The third class is geometric layout variables such as the length of spans in a continuous beam; and finally, cross section design variables such as the dimensions of a concrete section [22]. In most occasions, in optimization process, the designer is not free to set the pre-assigned parameters and design variables, and the existing circumstances may dictate to the designer what to do. Exploiting experience, nevertheless, one may shift from one set of variables to another that causes a new definition for the objective function (cost function in this case). Moreover, any changes in choosing design variables may lead to changes in constraints and even vary their nature from design variables to behaviour ones and vice versa. In other words, depending on the nature of the optimization problem, the process of achieving an optimum feasible solution may be much quicker, shifting from one design space to another by changing design variables as the space dimensions.

The selection of the cost function can be considered the most important decision in the entire optimal design process. Therefore, it is essential to introduce a cost function that represents the most influential cost components and more importantly, is applicable for a variety of similar optimization problems. Furthermore, it must be capable of matching the explicit constraints of structures, which are often given by formulas in design codes. 


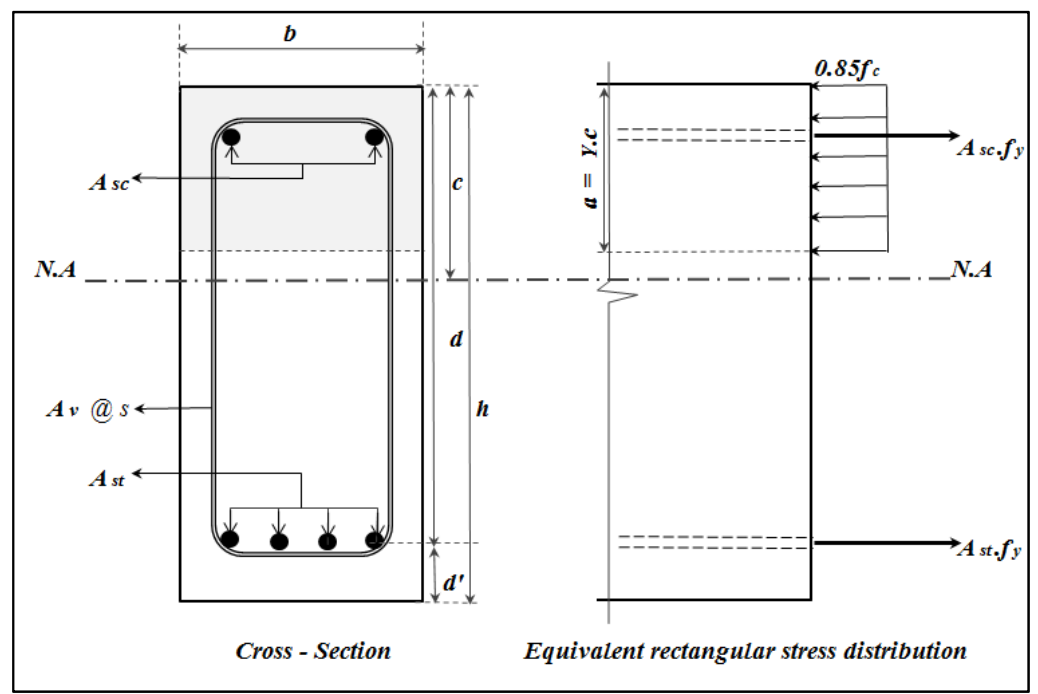

Figure 1: An arbitrary section of a rectangular $\mathrm{RC}$ beam $[10,9]$

A cost function generally includes the cost of materials, transportation, fabrication and even maintenance costs, in addition to repair and insurance costs, which can be presented by a weighted sum of a number of properties. The effect of these factors in optimal cost can be imposed on the weighted coefficients of the cost function. In concrete structures, at least three different cost items should be considered in optimization: costs of concrete, steel, and the formwork. So, the general cost function for a reinforced concrete beam can be expressed in the following form [10, 9]:

$$
C=C_{c}+C_{s}+C_{f}
$$

where $C, C_{c}, C_{s}$ and $C_{f}$ are the total cost, cost of concrete, cost of steel and cost of the formwork, respectively. Obviously, for pre-stressed and fibre reinforced concrete sections the relevant costs for these two items are added to the total cost. Considering the unit costs per parameter and the fact that, unit costs of longitudinal reinforcement steel usually differ from shear steel; Eq. (1) can be written as

$$
C=c_{c} A_{c}+c_{s l} A_{s l}+c_{s v} A_{s v}+c_{f} P_{f}
$$

Where $c_{c}, c_{s l}, c_{s v}$ and $c_{f}$ are the unit costs of concrete, longitudinal steel, shear steel and formwork respectively and $A_{c}, A_{s l}, A_{s v}$ and $P_{f}$ are their corresponding quantities. In most published optimization studies [23], the costs are calculated for a member such as a beam, and coefficients and quantities are presented based on members or the unit length of each member in Eq. (2). If such a classic cost function for a beam optimization problem is used, some or all of the parameters $A_{c}, A_{s l}, A_{s v}$ and $P_{f}$ are considered the design variables. Then, based on explicit constraints, which are presented in codes and/or implicit behavior constraints of the problem, the optimum values of the design variables will be found using optimization techniques. In other 
words, most existing cost optimization functions deal only with cross-sectional variables.

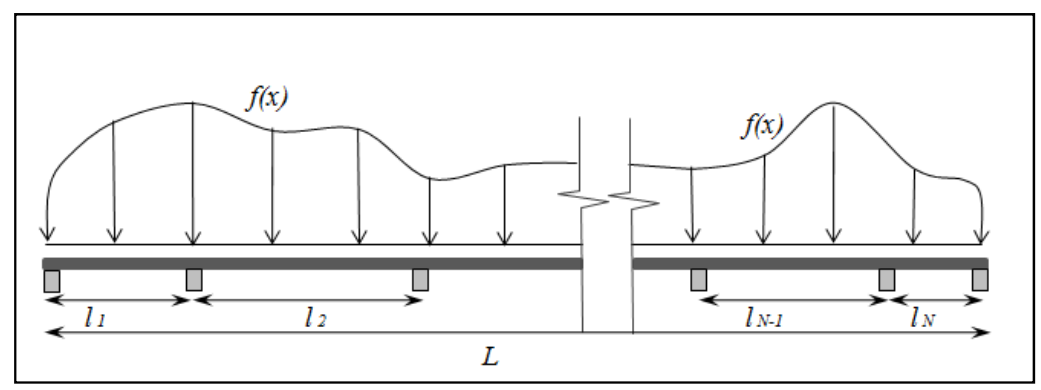

Figure 2: A multi-span rectangular RC beam

In case of the multiobjective optimization of structures including a combination of material, geometrical layout, topological and cross-sectional design variables, using currently available formulation leads to a significant number of design variables and constraints. This is because each of the stated conditions might require a complicated trial and error procedure. In fact, the design variables of this kind, even in the traditional methods of design, require sound engineering judgment to be chosen, and it can be extremely time-consuming to determine their solution for each section of each member. Therefore, unless alternative design variables are selected for the cost function, the multiobjective optimization procedure might be too unwieldy in such cases.

In the problem of preliminary layout optimization of multi-span RC beams, parameters like cross-sectional area, perimeter of concrete section or area of reinforcement steel are not truly suitable as design variables, because such variables are not obtained from an explicit mathematical procedure. These parameters are mainly calculated using suggested relations in design codes, which do not uniquely provide the exact values for these parameters. Furthermore, in order to determine such parameters, the first step is to complete the structural analysis procedure. Then, using the structural analysis outputs such as forces and moments together with design code requirements, the cross-sectional characteristics of the concrete beam are primarily determined. In such a case, each step of the layout optimization, as in an iterative procedure, includes both analysis and design processes, and besides the classic cross-sectional variables, the layout of a structure and consequently the outputs of structural analysis would be variables of the problem. Therefore, designers have to repeat the design procedure to achieve the optimal cross-sectional variables that are usually functions of other analysis outputs.

Consider Eq. (3) as a potential alternative cost function to Eq. (2) in an arbitrary beam cross-section.

$$
\mathrm{C}=\mathrm{c}_{1} M_{u}^{+}+\mathrm{c}_{2} M_{u}^{-}+\mathrm{c}_{3} \mathrm{~V}_{\mathrm{u}}
$$


where $M_{u}^{+}$and $M_{u}^{-}$are the positive and negative bending moment capacities and $V_{u}$ is the shear capacity of the section. If an appropriate set of $\left\{c_{1}, c_{2}, c_{3}\right\}$ could be found, such that Eq. (3) represents the cost of the section, the design variables would shift from $A_{c}, A_{s l}, A_{s v}$ and $P_{f}$ to $M_{u}^{+}, M_{u}^{-}$and $V_{u}$. In fact, due to the relationship between the capacity factors of the section and structural analysis outputs in design codes, the aim of using cost functions such as Eq. (3) is to use structural analysis outputs instead of structural design factors.

Using structural analysis outputs, say internal action effects of a beam, as design variables, has some advantages over using structural design outcomes such as crosssectional characteristics of a beam. Firstly, design action effects of each section can be easily obtained from structural analysis, and in an iterative mathematical procedure, re-analyzing a structure is considerably less time-consuming and more precise than re-designing the structure. If Eq. (2) is used for finding the optimum layout of a large structure with respect to the involved cost elements, the optimization tool needs to deal with both structural analysis and structural design in each step in order to move towards an optimum solution. Besides, in the process of obtaining $A_{c}, A_{s l}, A_{s v}$ and $P_{f}$, in the first step, one needs to have access to the relevant $M_{u}^{+}, M_{u}^{-}$and $V_{u}$. Therefore, using $M_{u}^{+}, M_{u}^{-}$and $V_{u}$ as optimization variables seems to be a shortcut in finding the optimum cost in the layout optimization of multi-span beams.

Moreover, in Eq. (3), the cost function is considered in a section rather than a member. Such ability is an advantage, as it enables the designer to select a number of sections for each member and in the entire structure to control the cost, and there is no necessity to conduct the optimization process over the entire member. Since the cross-sectional characteristics of a member, say a RC beam, such as $A_{c}, A_{s l}, A_{s v}$ and $P_{f}$ varies along the member, in order to come up with an accurate cost function for a member, one needs to have a clear idea about the probable distribution of bending moments and shear forces. Such a requirement makes the formulation extremely complicated and sometimes impractical for some multi-objective problems.

The rest of this paper describes how to find an appropriate set of coefficients that make Eq. (3) a viable alternative to Eq. (2) for layout optimization of reinforced concrete continuous beams. Using the proposed cost function, a method to find the optimum lengths of spans in a multi-span beam in order to minimize the cost is formulated.

\section{Mathematical Formulation}

In formulating the cost optimization of a beam and the relevant constraints, the Australian design standard for reinforced concrete structures, AS-3600 [24] is used, which is based on limit states design method of concrete structures. The evaluation of the area of steel reinforcement and the area of concrete section are based on the strength limit states of the section that can occur in either concrete or steel.

Consider an arbitrary section of a rectangular reinforced concrete beam as shown in Figure 1. The dimensions of the section are $b$ and $h$, the areas of tension and 
compression reinforcement steel are $A_{s t}, A_{s c}$ respectively and the area of shear reinforcement steel in a unit length of beam is $A_{s v} / s$.

The capacity or the ultimate strength of the section in negative and positive flexure and shear are $M_{u}^{-}, M_{u}^{+}$and $V_{u}$ respectively, which can be obtained from Eq. (4) through Eq. (6) in a balanced section in which both tension and compression steel yield $[9,10]$.

$$
\begin{gathered}
M_{u}^{-} \cong A_{s c} f_{y l} d\left(1-0.5 \gamma k_{u}\right) \\
M_{u}^{+} \cong A_{s c} f_{y l} d\left(1-0.5 \gamma k_{u}\right)+\left(A_{s t}-A_{s c}\right) f_{y l}(d-a / 2) \\
V_{u} \cong f_{y v} d A_{s v} / s+\beta b d\left(f_{c}^{\prime}\right)^{(1 / 2)}
\end{gathered}
$$

where $f_{y l}$ and $f_{y v}$ are the yield strength of the longitudinal reinforcement steel and the shear reinforcement steel respectively, $f_{c}^{\prime}$ is the characteristic compressive cylinder strength of concrete at 28 days, and $\beta$ is a coefficient based on the standard. Parameters $k_{u}$ and $\gamma$ are the neutral axis parameter and the ratio of the depth of the assumed rectangular compressive stress block to $k_{u} d$. Other parameters are shown in Figure 1.

In order to shift from Eq. (2) to Eq. (3) and come up with the set of $\left\{c_{1}, c_{2}, c_{3}\right\}$, the first step is to determine how variations of $A_{c}, A_{s l}, A_{s v}$ and $P_{f}$ affect $M_{u}^{+}, M_{u}^{-}$and $V_{u}$ and vice versa. That is, the reciprocal relationships between these two sets of variables need to be identified, and to be found out how increasing or decreasing the amount of each cross-sectional parameter influences the section strength capacities and how one should change the cross-sectional parameters to vary section capacities. As stated above, given the unit costs $c_{c}, c_{s l}, c_{s v}$ and $c_{f}$, the cost function can be defined using Eq. (2) for each section. If any of cross-sectional parameters $A_{c}, A_{s l}$, $A_{s v}$ or $P_{f}$ changes, the cost function varies in a section as follows

$$
\Delta C=c_{c} \Delta A_{c}+c_{s l} \Delta A_{s l}+c_{s v} \Delta A_{s v}+c_{f} \Delta P_{f}
$$

On the other hand, using Eq. (3) variations in section capacities would change the cost function as follows

$$
\Delta C=c_{1} \Delta M_{u}^{+}+c_{2} \Delta M_{u}^{-}+c_{3} \Delta V_{u}
$$

Eqs. (7) and (8) show the contribution of each factor to cost changes and sensitivity of the cost to each term. For example, changing a unit of $A_{c}$ causes a change of $c_{c}$ units in cost. Therefore, if the effect of variations of $A_{c}, A_{s l}, A_{s v}$ and $P_{f}$ on variations of $M_{u}^{+}, M_{u}^{-}$and $V_{u}$ are determined, the contribution of each section capacity to cost changes, that is the set of $\left\{c_{1}, c_{2}, c_{3}\right\}$, can be found out.

In order to simplify the calculations, two assumptions are made. First, since we intend the section to remain in a balanced condition, any changes for compression steel are considered equal to those of tension steel.

$$
\Delta A_{s t}=\Delta A_{s c}=\Delta A_{s l}
$$


This means, in case of the layout optimization of multi-span beams in which, the aim is to find the optimum spans lengths, variation of steel quantities for compression and tension steel in a balanced section can be considered equal in each step of an iterative process. Such an assumption causes a section to remain in balanced condition in every step and results in a balanced section at the end of the optimization procedure. The second assumption is that the depth of the compressive region of concrete, the parameter $a$, is not considerably affected by variations of the section width $b$. The width of a beam section is generally constrained by a number of factors including architectural constraints, standards' limitations and limitations of columns and joints. All these constraints cause the width of the section to have a minor variation during the optimization process. It does not mean that the variation of width is ignored, but in practice, such a variation is so limited that it has a negligible effect on the depth of the compressive region.

Considering the fore mentioned assumptions and using Eqs. (4) through (7), if $A_{s}$ varies:

$$
\left\{\begin{array}{l}
\frac{\Delta M_{u}^{-}}{\Delta A_{s l}} \cong f_{y l} d\left(1-0.5 \gamma k_{u}\right) \rightarrow \Delta A_{s l} \cong\left[f_{y l} d\left(1-0.5 \gamma k_{u}\right)\right]^{-1} \Delta M_{u}^{-}=K_{1} \Delta M_{u}^{-} \\
\frac{\Delta M_{u}^{+}}{\Delta A_{s l}} \cong f_{y l} d\left(1-0.5 \gamma k_{u}\right) \rightarrow \Delta A_{s l} \cong\left[f_{y l}\left(1-0.5 \gamma k_{u}\right)\right]^{-1} \Delta M_{u}^{+}=K_{1} \Delta M_{u}^{+} \\
\frac{\Delta V_{u}}{\Delta A_{s l}} \cong 0 \rightarrow \Delta V_{u} \text { and } \Delta A_{s l} \text { are indipendant of each other }
\end{array}\right.
$$

If $A_{v}$ varies, the variation of the section capacity will be as follow:

$$
\left\{\begin{array}{l}
\Delta M_{u}^{-} \text {and } \frac{\Delta A_{s v}}{s} \text { are indipendant of each other } \\
\Delta M_{u}^{+} \text {and } \frac{\Delta A_{s v}}{s} \text { are indipendant of each other } \\
\frac{\Delta V_{u}}{\left(\frac{\Delta A_{s v}}{s}\right)} \cong f_{y v} d \rightarrow \frac{\Delta A_{s v}}{s} \cong\left(f_{y v} d\right)^{-1} \Delta V_{u}=K_{2} \Delta V_{u}
\end{array}\right.
$$

If the area of section, i.e. $b d$ varies:

$$
\Delta A_{c}=\Delta(b d)=b \Delta d+d \Delta b+\Delta b \Delta d
$$

$M_{u}^{-}$is not a function of $b$ and based on the second assumption we made, the dependence of $M^{+}{ }_{u}$ on changes of $b$ is neglected. Moreover, the first term of $V_{u}$ is not dependent on $b$ as well. So, the variations of the section capacities due to variation of section area are as follow:

$$
\frac{\Delta M_{u}^{(b)}}{\Delta A_{c}^{(b)}}=\frac{\Delta M_{u}^{(b)}}{\Delta(b d)} \cong \frac{\Delta M_{u}^{(b)}}{b(\Delta d)}
$$




$$
\left\{\begin{array}{c}
\frac{\Delta M_{u}^{-}}{\Delta(b d)} \cong \frac{f_{y l} A_{s c}}{b}\left(1-0.5 \gamma k_{u}\right) \rightarrow \Delta(b d) \cong\left[f_{y l} \frac{A_{s c}}{b}\left(1-0.5 \gamma k_{u}\right)\right]^{-1} \Delta M_{u}^{-}=K_{3} \Delta M_{u}^{-} \\
\frac{\Delta M_{u}^{+}}{\Delta(b d)} \cong \frac{f_{y l}}{b}\left(A_{s t}-0.5 \gamma k_{u} A_{s c}\right) \rightarrow \Delta(b d) \cong\left[\frac{f_{y l}}{b}\left(A_{s t}-0.5 \gamma k_{u} A_{s c}\right)\right]^{-1} \Delta M_{u}^{+}=K_{4} \Delta M_{u}^{+} \\
\frac{\Delta V_{u}}{\Delta(b d)} \cong \frac{f_{y v} A_{s v}}{b s}+\beta\left(f^{\prime}{ }_{c}\right)^{1 / 2} \rightarrow \Delta(b d) \cong\left[\frac{f_{y v} A_{s v}}{b s}+\beta\left(f^{\prime}{ }_{c}\right)^{1 / 2}\right]^{-1} \Delta V_{u}=K_{5} \Delta V_{u}
\end{array}\right.
$$

The variation of the perimeter of a rectangular section, which determines the variation of formwork, affects the section capacity as follow:

$$
\begin{aligned}
& \Delta P_{f}=\Delta b+2 \Delta d \\
& \left\{\begin{array}{l}
\frac{\Delta M_{u}}{\Delta b+2 \Delta d} \cong 0.5 f_{y l} A_{s c}\left(1-0.5 \gamma k_{u}\right) \rightarrow \Delta P_{f} \cong 2\left[f_{y l} A_{s c}\left(1-0.5 \gamma k_{u}\right)\right]^{-1} \Delta M_{u}^{-}=K_{6} \Delta M_{u}^{-} \\
\frac{\Delta M_{u}^{+}}{\Delta b+2 \Delta d} \cong 0.5 f_{y l}\left(A_{s t}-0.5 \gamma k_{u} A_{s c}\right) \rightarrow \Delta P_{f} \cong 2\left[f_{y l}\left(A_{s t}-0.5 \gamma k_{u} A_{s c}\right)\right]^{-1} \Delta M_{u}^{+}=K_{7} \Delta M_{u}^{+} \\
\frac{\Delta V_{u}}{\Delta b+2 \Delta d} \cong \frac{2}{\frac{f_{y l} l_{s v}}{s}+\beta b\left(f^{\prime}{ }^{\prime}\right)^{1 / 2}}+\frac{1}{\beta d\left(f^{\prime}{ }^{\prime}\right)^{1 / 2}} \rightarrow \Delta P_{f} \cong\left[\frac{2}{\frac{f_{y v A_{s v}}}{s}+\beta b\left(f^{\prime} c^{\frac{1}{2}}\right.}+\frac{1}{\beta d\left(f^{\prime}{ }^{\prime}\right)^{\frac{1}{2}}}\right] \Delta V_{u}=K_{8} \Delta V_{u}
\end{array}\right.
\end{aligned}
$$

Multiplying both sides of Eqs. (10) by $c_{s l} / 2$, Eqs. (11) by $c_{s v}$, Eqs. (14) by $c_{c} / 3$ and Eqs. (16) by $c_{f} / 3$, and adding them up will result in:

$$
\begin{aligned}
c_{c} \Delta A_{c}+c_{s l} \Delta A_{s l}+c_{s v} \Delta A_{s v}+c_{f} \Delta P_{f}= & \left(\frac{1}{3} c_{c} K_{4}+\frac{1}{2} c_{s l} K_{1}+\frac{1}{3} c_{f} K_{7}\right) \Delta M_{u}^{+} \\
& +\left(\frac{1}{3} c_{c} K_{3}+\frac{1}{2} c_{s l} K_{1}+\frac{1}{3} c_{f} K_{6}\right) \Delta M_{u}^{-} \\
& +\left(\frac{1}{3} c_{c} K_{5}+c_{s v} K_{2}+\frac{1}{3} c_{f} K_{8}\right) \Delta V_{u}
\end{aligned}
$$

Comparing Eq. (17) with Eqs. (8) and (9) results in:

$$
\left\{\begin{array}{l}
c_{1}=\frac{1}{3} c_{c} K_{4}+\frac{1}{2} c_{s l} K_{1}+\frac{1}{3} c_{f} K_{7} \\
c_{2}=\frac{1}{3} c_{c} K_{3}+\frac{1}{2} c_{s l} K_{1}+\frac{1}{3} c_{f} K_{6} \\
c_{3}=\frac{1}{3} c_{c} K_{5}+c_{s v} K_{2}+\frac{1}{3} c_{f} K_{8}
\end{array}\right.
$$

The coefficients $\mathrm{c}_{1}, \mathrm{c}_{2}$ and $\mathrm{c}_{3}$ determine how the parameters $M_{u}^{-}, M_{u}^{+}$and $V_{u}$ contribute to the cost function. Now, in order to re-analyze a rectangular beam to achieve the optimum criteria, one can use Eq. (3) in lieu of Eq. (2). For this purpose, the cost will be the sum of cost functions of all selected sections in the structure.

$$
C_{t}=\sum_{i=1}^{N S} C_{i}=\sum_{i=1}^{N S}\left(c_{1 i} M_{u i}^{+}+c_{2 i} M_{u i}^{-}+c_{3 i} V_{u i}\right)
$$

where $C_{t}$ is the total cost of the structure and NS is the number of selected sections to control the cost.

The strength constraints on each selected section $i$ in the structure under a load case may be written as 


$$
\left\{\begin{array}{l}
\emptyset_{f} M_{u i}^{+} \geq M_{i}^{*^{+}} \\
\emptyset_{f} M_{u i}^{-} \geq M_{i}^{*^{-}} \\
\emptyset_{s} V_{u i} \geq\left|V_{i}{ }^{*}\right|
\end{array} \quad \text { for } \mathrm{i}=\{1,2, \ldots \mathrm{NS}\}\right.
$$

in which, $M_{i}{ }^{{ }^{+}}, M_{i}{ }^{{ }^{-}}$and $\left|V_{i}{ }^{*}\right|$ are positive and negative flexure and shear action effects of section $i . \emptyset_{f}$ and $\emptyset_{s}$ are strength reduction factors in flexure and shear respectively. The serviceability requirements limit the maximum deflection $u_{\max }$ on the entire member to $\Delta_{\max }$ under the serviceability load case. So, for all sections:

$$
\mathrm{u}_{\max \mathrm{i}} \leq \Delta_{\max \mathrm{i}} \quad \text { for } \mathrm{i}=\{1,2, \ldots \mathrm{NS}\}
$$

Other constraints for durability, fire resistance, minimum cover and minimum flexural strength, can be easily added to the problem as well, based on the relevant design codes.

Now, consider a multi-span RC beam with $N$ spans and a total constant length of $L$, under the arbitrary loading system $f(x)$ as shown in Figure 2. The aim is to re-design the beam to determine the optimum span lengths in order to minimize the cost. According to Eqs. (19) and (20), the total cost is a function of the beams' action effects under the loading system, which in turn are functions of the span lengths in a structural analysis. The general formulation of the problem is:

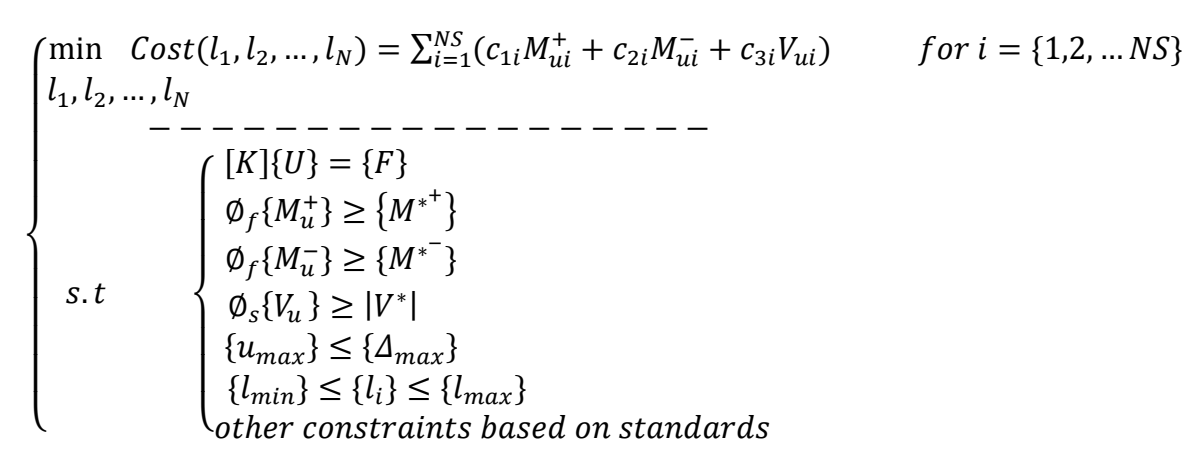

where the first constraint is the behavior constraint of the structural optimization problem determining the relation between design action effect $\left\{M^{*^{+}}\right\},\left\{M^{*^{-}}\right\}$and $\left\{V^{*-}\right\}$, and spans lengths based on general formulation of the displacement method in which, $[K]$ is the global stiffness matrix of the structure, $\{U\}$ is the displacement vector and $\{F\}$ is the externally load vector obtained from $f(x)$. Using the EulerBernoulli beam element for finite element analysis of structures, the dimension of the above vectors will be $2 N$; where $N$ is the number of spans. The dimension of other variable constraint vectors, say $\left\{M_{u i}^{+}\right\},\left\{M_{u i}^{-}\right\}$, is $N S$; because the other constraints and cost function will be evaluated in $N S$ critical sections. Furthermore, the length of each span can be constrained between $l_{\min }$ and $l_{\max }$ in order to satisfy architectural considerations $[9,10]$. 


\section{Numerical Examples}

Example 1: consider a three-span beam with geometry details, live, and dead loads shown in Figure 3. The beam supports a slab of thickness $120 \mathrm{~mm}$ in the interior of a residential building. The material properties and relative cost factors are: $f_{c}^{\prime}=25$ $\mathrm{MPa}, f_{y l}=f_{y v}=400 \mathrm{MPa}, c_{c}=1, c_{s l}=c_{s v}=66, c_{f}=0.42$. The ratio of effective span to the maximum total deflection should not be less than 250 . The aim is to find the optimum $l_{1}, l_{2}$, and $l_{3}$, so that the cost is minimized.

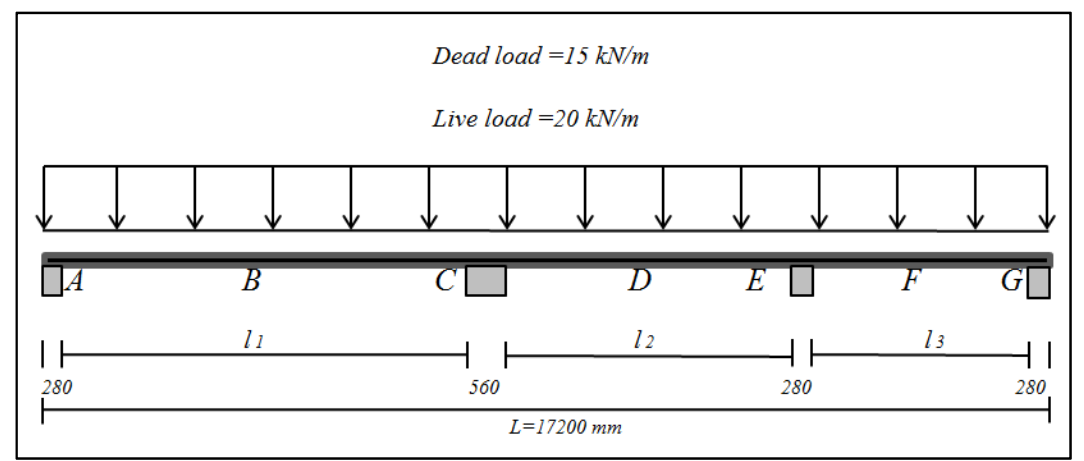

Figure 3: Example 1- A three-span beam with uniformly distributed loads

A similar problem given $l_{1}=7000 \mathrm{~mm}, l_{2}=5000 \mathrm{~mm}$ and $l_{3}=4000 \mathrm{~mm}$, has been solved by Kanagasunda and Karihaloo [25]. This design is used as the initial design to start the optimization process for the spans. It should be added that every initial design based on preliminary judgment of the designer and/or using approximate charts or formulas, which meet the design code requirements, can be used as the initial design and as the starting point of optimization process. According to their solution for the seven selected sections A through G, based on AS3600 (2009), and considering the abovementioned costs, the primary cost of the structure based on Eq. (2) equals 4648 units

It should be noted that, the number of selected sections completely depends on the number of critical or control ones, and one may choose more sections to achieve much accurate results. Using Eqs. (10), (11), (14) and (16) the values of $K_{1}$ to $K_{8}$, and using Eq. (18) the values of $c_{1}, c_{2}$ and $c_{3}$ for sections $A$ to $G$ are obtained. Having the necessary coefficients, the cost function can be defined, based on Eq. (19).

The optimum lengths are $l_{1}=4740 \mathrm{~mm}, l_{2}=5980 \mathrm{~mm}$ and $l_{3}=5280 \mathrm{~mm}$ resulting in a total cost of 3817 units based on Eq. (2). That means the recent layout with the obtained spans lengths suggest around $18 \%$ less cost compared to the initial layout. 


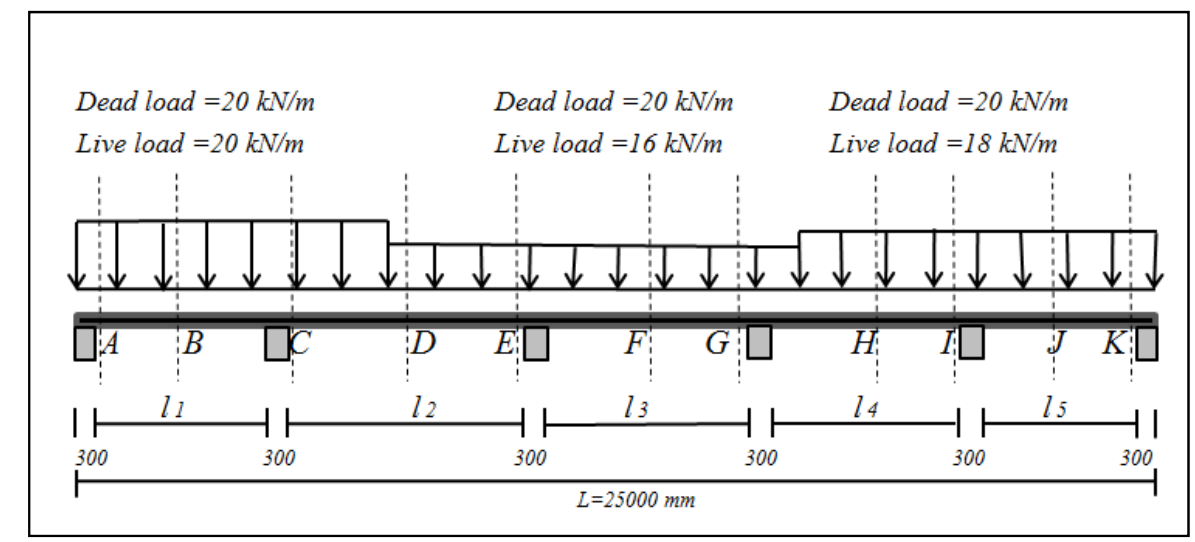

Figure 4: Example 2 - A five span beam and selected sections to control the cost

Example 2: A five-span continuous beam with a total length of $25000 \mathrm{~mm}$ is considered as shown in Figure 4. The material properties and relative cost factors are: $f_{c}^{\prime}=25 \mathrm{MPa}, f_{y l}=500 \mathrm{MPa}, f_{y v}=250 \mathrm{MPa}, c_{c}=1, c_{s l}=75, c_{s v}=64, c_{f}=0.45$. The aim is to find the optimum $l_{1}$, through $l_{5}$, so that the cost is minimized. The eleven sections A through $\mathrm{K}$, are taken as the control sections.

The preliminary design of the beam comprised five equal spans, which results in a total cost of 5949 units. The optimum lengths are $l_{1}=3450 \mathrm{~mm}, l_{2}=4600 \mathrm{~mm}, l_{3}$ $=5400 \mathrm{~mm}, l_{4}=4400 \mathrm{~mm}$ and $l_{5}=5350 \mathrm{~mm}$ resulting in a total cost of 5220 units.

\section{Conclusion}

The main objective of this study is to propose an appropriate model for cost optimization of RC beams layout design. This model considers the effects of layout design on total cost, and suggests an optimization method for layout design of multispan beams. Using action effects instead of cross-sectional properties of structures, the proposed model simplifies the process of cost and layout optimization of multispan beams, and is applicable to various topology optimization problems of RC beams when the cost elements are considered. The presented examples show that the proposed algorithm using the new cost optimization function provides acceptable results, and can be easily employed to optimally design the preliminary layout of RC continuous beams.

\section{References}

1. R.D. Rush, The Building systems integration handbook. American Institute of Architects: Butterworth-Heinemann. 1986, 
2. S.A. Burns, Recent advances in optimal structural design: American Society of Civil Engineers.Structural Engineering Institute. Technical Committee on Optimal Structural Design. 2002,

3. G. Rozvany, A critical review of established methods of structural topology optimization. Structural and Multidisciplinary Optimization, 37(3): p. 217237. 2009.

4. G.I.N. Rozvany, N. Olhoff, Topology optimization of structures and composite continua: Kluwer Academic Publishers. 2000,

5. G.I.N. Rozvany, I.C.f.M. Sciences, Topology Optimization in Structural Mechanics: Springer. 1997,

6. A. Kaveh, P. Sharafi, Ant colony optimization for finding medians of weighted graphs. Engineering Computations, 25(2): p. 102-120. 2008.

7. P. Sharafi, M.N.S. Hadi, L.H. Teh, Optimum Column Layout Design of Reinforced Concrete Frames Under Wind Loading Topics on the Dynamics of Civil Structures, Volume 1, J.M. Caicedo, et al., Editors, Springer US. p. 327-340. 2012

8. P. Sharafi, M.N.S. Hadi, L.H. Teh, Optimum Spans' Lengths of Multi-span Reinforced Concrete Beams Under Dynamic Loading Topics on the Dynamics of Civil Structures, Volume 1, J.M. Caicedo, et al., Editors, Springer US. p. 353-361. 2012

9. P. Sharafi, M.N.S. Hadi, L.H. Teh, Geometric Design Optimization for Dynamic Response Problem of Continuous Reinforced Concrete Beams. ASCE Journal of Computing in Civil Engineering, In Press. 2012.

10. P. Sharafi, M.N.S. Hadi, L.H. Teh, Heuristic Approach for Optimum Cost and Layout Design of 3D Reinforced Concrete Frames. Journal of Structural Engineering, 138(7): p. 853-863. 2012.

11. P. Sharafi, M.N.S. Hadi, L.H. Teh, Cost Optimization of Column Layout Design of Reinforced Concrete Buildings, in Metaheuristic Applications in Structures and Infrastructures A. Gandomi, et al., Editors, Elsevier: London. 2013

12. V. Govindaraj, J.V. Ramasamy, Optimum detailed design of reinforced concrete continuous beams using Genetic Algorithms. Computers \& Structures, 84(1-2): p. 34-48. 2005.

13. A. Muc, W. Gurba, Genetic algorithms and finite element analysis in optimization of composite structures. Composite Structures, 54(2-3): p. 275281. 2001.

14. J.G. Wang, G.R. Liu, On the optimal shape parameters of radial basis functions used for 2-D meshless methods. Computer Methods in Applied Mechanics and Engineering, 191(23-24): p. 2611-2630. 2002.

15. M.N.S. Hadi, Neural networks applications in concrete structures. Computers \& Structures, 81(6): p. 373-381. 2003.

16. A. Nimtawat, P. Nanakorn, Automated layout design of beam-slab floors using a genetic algorithm. Computers \& Structures, 87(21-22): p. 1308-1330. 2009.

17. A. Nimtawat, P. Nanakorn, A genetic algorithm for beam-slab layout design of rectilinear floors. Engineering Structures, 32(11): p. 3488-3500. 2010. 
18. J. Zhu, W. Zhang, P. Beckers, Y. Chen, Z. Guo, Simultaneous design of components layout and supporting structures using coupled shape and topology optimization technique. Structural and Multidisciplinary Optimization, 36(1): p. 29-41. 2008.

19. D. Shaw, J. Miles, A. Gray, Determining the structural layout of orthogonal framed buildings. Computers \& Structures, 86(19-20): p. 1856-1864. 2008.

20. X.K. Zou, C.M. Chan, G. Li, Q. Wang, Multiobjective Optimization for Performance-Based Design of Reinforced Concrete Frames. Journal of Structural Engineering, 133(10): p. 1462-1474. 2007.

21. S. Liu, H. Qiao, Topology optimization of continuum structures with different tensile and compressive properties in bridge layout design. Structural and Multidisciplinary Optimization, 43(3): p. 369-380. 2011.

22. U. Kirsch, Optimum structural design: concepts, methods, and applications: McGraw-Hill. 1981,

23. H. Adeli, K. Sarma, Cost optimization of structures: fuzzy logic, genetic algorithms, and parallel computing: Wiley. 2006,

24. AS3600, Concrete structures. Standards Association of Australia: Sydney. 2009,

25. S. Kanagasundaram, B.L. Karihaloo, Minimum-cost design of reinforced concrete structures. Computers \&amp; Structures, 41(6): p. 1357-1364. 1991. 